\title{
The Analysis of the Noise Generation in Gas Turbine Stage
}

\author{
Sławomir Dykas, Dawid Machalica \\ Institute of Power Engineering and Turbomachinery, Silesian University of Technology, Gliwice, Poland \\ Email: slawomir.dykas@polsl.pl
}

Received 14 September 2014; revised 13 October 2014; accepted 13 November 2014

Copyright (C) 2014 by authors and Scientific Research Publishing Inc.

This work is licensed under the Creative Commons Attribution International License (CC BY). http://creativecommons.org/licenses/by/4.0/

(c) (i) 0pen Access

\begin{abstract}
The aim of this paper is to assess the impact of the mutual positioning of the turbine stage stator and rotor blades on noise generation. The Ansys CFX commercial software package and the Scale-Adaptive Simulation (SAS) hybrid turbulence model are used for numerical analyses. The paper is focused on an analysis that the pressure wave generation resulting from unsteady flow phenomena. In order to present the problem, the Fast Fourier Transformation (FFT) analysis of pressure fluctuation is carried out at selected points of the turbine stage computational domain. A comparison of values of individual components for subsequent control points allows an approximate determination of the place of generation of pressure waves, the direction of their propagation and the damping rate. Moreover, the numerical analyses make it possible to evaluate the justification for the use of the SAS model, which is rather demanding in terms of equipment, in simulations of unsteady flow fields where generation and propagation of noise waves occur.
\end{abstract}

\section{Keywords}

Turbine Stage, Noise, FFT Analysis

\section{Introduction}

The rapid development of numerical methods and techniques in the last two decades has made it possible to carry out more and more complex analyses using the Computational Fluid Dynamics (CFD) tools. Owing to the use of computer software in flow analyses, it is possible to avoid high costs related to the construction of test stations and reduce the time needed to obtain the optimum structure. It is also significant that simulation results contain full information on the flow medium properties which under experimental conditions often cannot be measured (e.g. density or entropy). These advantages contribute to the fact that numerical analyses are gaining popularity in industries such as power engineering or aviation. This, however, does not mean that experimental 
testing should be discontinued as it is necessary to validate numerical models.

A correct assessment of the turbine stage operation should be made through an analysis of unsteady phenomena which provides lots of information concerning instantaneous distributions of pressures and other flow parameters of the medium. The knowledge of these parameters is useful while analysing vibration or acoustic phenomena. In extreme situations they may lead to destruction of the machine components due to high cycle wear. The knowledge of pressure fluctuation at individual points of the blade channel makes it also possible to perform analyses of generation and propagation of pressure waves, including acoustic waves (noise or/and vibrations). Nowadays, this is of special importance considering the ever-stricter restrictions on the noise level of, among others, aircraft engines. Because of the binding standards, manufacturers need to optimise the structure in terms of noise generation as early as at the designing stage. Only then will customers become interested in the supplier's products. Unsteady flow field analyses also allow, for example, an assessment of operation during a change in load. This gives an idea of the phenomena occurring inside the machine which quite often cannot be analysed experimentally.

The unsteady phenomena taking place in channels of turbomachines in many cases result in energy dissipation. Any phenomenon leading to a drop in efficiency is a loss [1]. Analysis of the losses in turbine stages by means various loss coefficients was performed by many authors [1]-[4]. However, the process of the acoustic wave generation in the flow field may also be perceived as a measure of energy dissipation.

This paper presents the methodology and the results of CFD analyses comprising the flow in the turbine stage of an engine. The topic was covered fairly often [5] [6]. However, the authors focused on selected issue only. For this purpose, the Ansys CFX commercial software is used. Due to anticipated complexity of the flow field, a decision was made to employ the Scale-Adaptive Simulation (SAS) hybrid turbulence model. This model can adapt itself dynamically to the already solved vortex street [7] [8]. Owing to that, it works perfectly in separation areas for example. In the paper, the focus is on the FFT analysis of pressure fluctuation at selected points of the computational domain. Comparing the values of varying pressure, it is possible to roughly determine the places of generation of acoustic waves, the direction in which they propagate and their damping rate.

\section{Analyzed Turbine Stage}

The object selected for the numerical analyses whose results are presented in this paper is the stage of a gas turbine intended for the aircraft industry [9] [10]. This turbine features a single stage only. A reduction in the number of stages involves a rise in the thermal and pressure drop per a turbine stage. This has an impact on the strength and flow properties. Moreover, the turbine under consideration was to operate ultimately at the temperature of the flue gases supplied to the stator at the level of $2200 \mathrm{~K}$. Such high temperature, unusual even in modern structures, required on part of the designers a development of cooling systems that would prevent blades from melting. In this paper, however, the issues related to blade cooling are ignored to reduce the size of the numerical mesh and to shorten the time of computations.

The analysed turbine is composed of $3638.1 \mathrm{~mm}$ high stator blades located at an average radius of $469.9 \mathrm{~mm}$ and 64 rotor blades with the same height and radius of location. The computations are performed for one stator channel and two rotor channels. Due to the lack of data concerning the geometry of the tip seal of the rotor blades, an example typical geometry is selected corresponding to two fins and a clearance of $0.3 \mathrm{~mm}$. The geometry of the stage under analysis is presented in Figure 1.

\section{Physical and Numerical}

The numerical analyses in this paper are carried out using the Ansys CFX commercial package using the uRANS method and the Scale-Adaptive Simulation (SAS) turbulence model. This model belongs to the group of hybrid models. It can adapt itself dynamically to the already solved vortex structures yielding good results in areas with intense swirling. It thus constitutes a compromise between the classic viscosity model and the DES or LES methods, which are rather costly in terms of computations and which are also available in the Ansys CFX package [4] [6].

The unsteady computations comprised almost 2000 time steps distanced from each other by $10^{-5} \mathrm{~s}$. This made it possible to carry out FFT analyses using 1024 samples, excluding the beginning stage steps which comprise the time for the solution to stabilize. Additionally, a short analysis was performed with a time step of $3 \times 10^{-6} \mathrm{~s}$, which allows the assessment of the changes in the values of loss coefficients depending on the position of the 


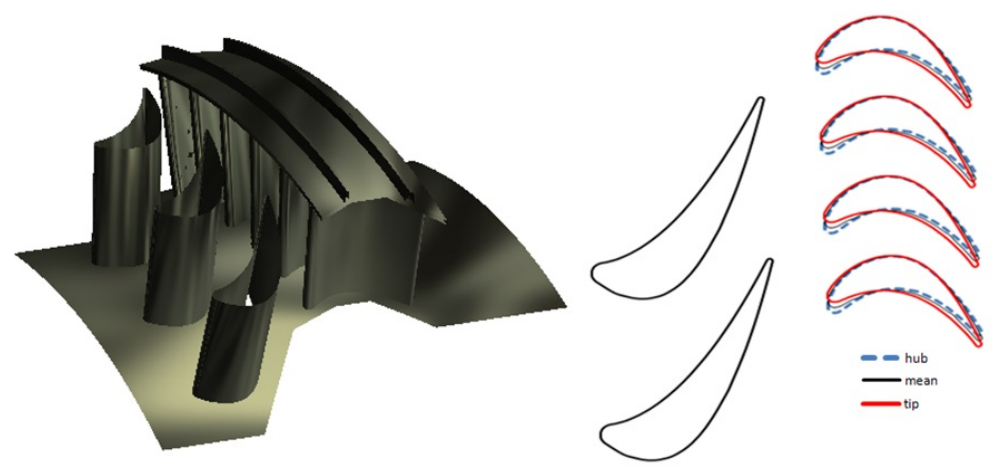

Figure 1. Geometry of the analysed turbine stage.

stator and rotor blades.

The desire to model the turbine stage together with the rotor blade tip seal requires a division of the computational area into three parts: the stator channel, the rotor channel and the seal area. For each of these parts a separate numerical mesh was generated. This accelerated the process of mesh generation and improved the mesh quality. The meshes for the flow channel around the stator and the rotor are of the mixed type, where the maximum value of the non-dimensional parameter $y^{+}$describing the size of the mesh near-wall element was approximately 1.20 .

The seal mesh was generated in the ICEM CFD program as fully structured. In order to improve the accuracy of transition modeling at the stator/rotor interface, two rotor channels were assumed for the calculations, which in total gave more than 4.7 M control volumes of the mesh for the entire stage with the seal (Figure 2).

The numerical analysis was carried out for the turbine design parameters described in a NASA report [10]. The characteristic feature of the turbine is that it has just one stage, which distinguishes it from other turbines used in turbofan engines. In order to achieve a required load the proposed value of the total temperature was $2200 \mathrm{~K}$, what is not reachable till nowadays. For reasons of simplicity, the medium fed to the stage is treated as perfect gas. The remaining boundary conditions used in the analysis are presented in Table 1.

\section{Numerical Results-FFT Analysis}

An FFT analysis was carried out at selected points of the computational area in order to estimate the places where pressure waves are generated and the direction in which they propagate. The location of the points discussed in this paper is presented in Figure 3.

The first element of the name of the control point Ro/U (Rotor/Seal) identifies the computational domain where the point is located. The element in/out/o identifies the axial location of the point with respect to the blade. The element b/ch indicates whether the point is located before the leading edge/in the vortex after the blade (b) or at the blade channel inlet/outlet (ch). This particular arrangement is to make it possible to estimate the distribution of pressure fluctuation in the axial and circumferential directions. Moreover, the points are located at three heights of $20 \%, 50 \%$ and $80 \%$ of the relative height of the blade channel.

\subsection{Ahead of the Rotor}

For points located before the rotor blade (Figure 4(a)), in the vicinity of the impact point, components p' feature quite similar amplitudes. The highest value of p' for the $13.2 \mathrm{kHz}$ component occurred in the bottom part of the channel; the lowest - in its middle part, where the effect of the walls restricting the flow is the smallest. It is also worth noting that despite the turbine very high rotational speed, the generated components with a high amplitude are still included in the audible frequency range.

The situation is identical at the rotor channel inlet (Figure 4(b)), where the distribution of p' amplitudes is the same as before the impact point. However, at the rotor channel inlet the amplitude values are higher by about 20 $\mathrm{kPa}$. The increase in amplitudes inside the channel is probably caused by the interference of waves arising at impact points of the blades located nearby.

In the case of points located before the rotor blade, apart from the $13.2 \mathrm{kHz}$ component, there is also a component with $\mathrm{f} \approx 8.6 \mathrm{kHz}$, and more components corresponding to it with frequencies increasing by approx- 


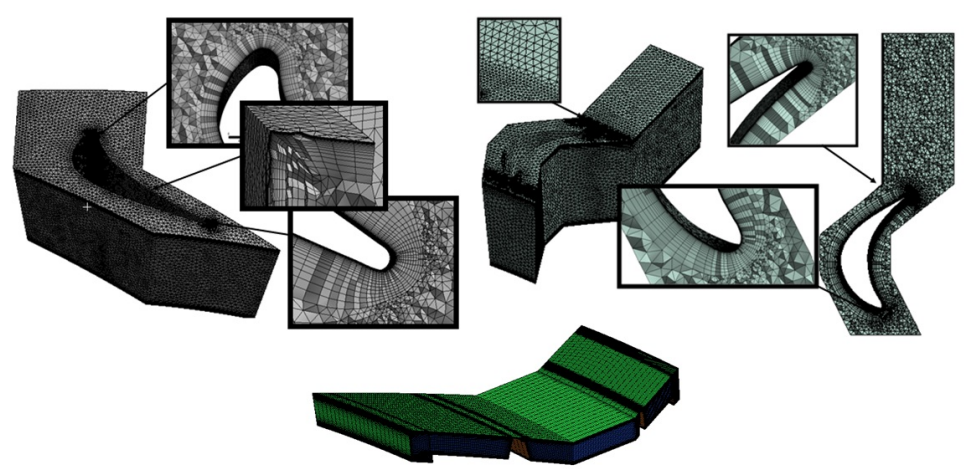

Figure 2. Computational meshes of the stator and rotor channels and of the seal.

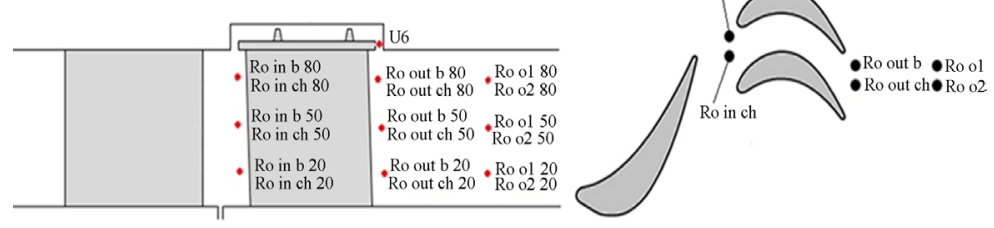

Figure 3. Arrangement of control points in the computational area.

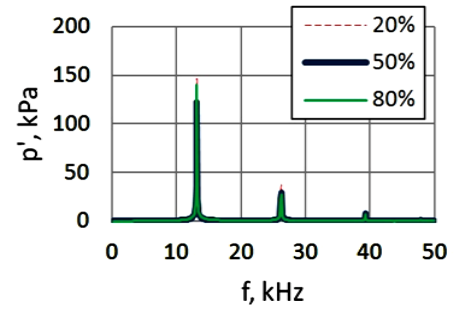

(a)

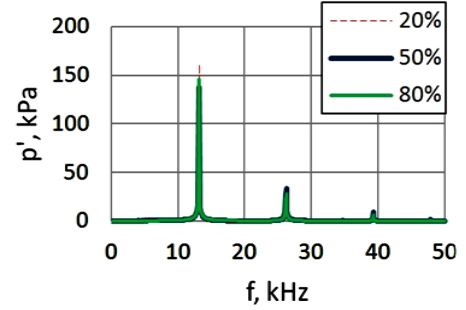

(b)

Figure 4. FFT analysis results for control points located before: (a) The rotor blade (points “Ro in b”); (b) The blade channel inlet (points “Ro in ch”).

Table 1. Data assumed for the real analysis.

\begin{tabular}{ccc}
\hline & & \\
\hline & Medium & Unsteady analysis \\
Turbulence model & Air as perfect gas \\
& Rotor rotational speed & SAS \\
& Stator/rotor interface type & $21,772 \mathrm{RPM}$ \\
Inlet & Total pressure & Transient rotor-stator \\
& Static temperature & $3.861 \mathrm{MPa}$ \\
Turbulence intensity & $2200 \mathrm{~K}$ \\
& Static pressure & $5 \%$ \\
\hline
\end{tabular}

imately the Vane Passing Frequency (VPF), which in this case is $\sim 13 \mathrm{kHz}$. It becomes especially visible if p' on the y axis is substituted with SPL (Figure 5). Here, each subsequent component features a lower amplitude reaching as much as $160 \mathrm{~dB}$ for $\mathrm{f}=47.9 \mathrm{kHz}$. 


\subsection{Seal Outlet}

The medium flow through the seal is extremely complex. The consequences of the very turbulent nature of the flow in this area can be observed especially at the outlet, where point U6 is located. The FFT analysis carried out for control point U6 (Figure 6) confirms this observation. The SAS model made it possible to capture a large number of new components than others two-equation eddy-viscosity turbulence models (for example the Shear Stress Transport (SST) model), including very significant ones with frequencies of $0.8 \mathrm{kHz}, 3.9 \mathrm{kHz}$ and $7.6 \mathrm{kHz}$.

The components appearing in the range of high frequencies may have their source both in the occurrence in the flow of vortex structures featuring small time scales and in the generation of acoustic waves arising due to the thermoacoustic effect. They would be induced by the temperature difference between the medium flowing out of the necking in the seal (over the fin) and the medium with a different temperature included in the seal cavity.

\subsection{Points Located before the Rotor Blade Inflow Edge and after the Blade, in the Aerodynamic Wake}

Comparing the results obtained for points Ro in b 80 and Ro out b 80 (Figure 7), a distinct reduction in the amplitude fluctuation by the VPF can be noticed for the point located after the blade. The reduction is almost 80 -fold. It can also be seen that after the blade, at the height of $80 \%$, a component can be detected with a frequency of $3.9 \mathrm{kHz}$, which is characteristic of the medium leaving the seal. It reaches $\sim 400 \mathrm{~Pa}$ and is thus about eight times smaller than at the seal outlet.

\subsection{Points Located after the Rotor Row}

After the rotor blade row, both at points located in the aerodynamic wake (Figure 8) and at the rotor channel outlet (Figure 9), amplitude p' for the $13.2 \mathrm{kHz}$ component diminishes with the blade channel height. This change is about fivefold for points located at the rotor channel outlet and 40-fold for points in the blade wake.

In the range of low frequencies, the components which are discernible first of all are those coming from the seal $(3.9 \mathrm{kHz})$, which are present only at control points located at $80 \%$ of the channel height. The amplitude of a component featuring this frequency is three times higher at a point after the blade than at the blade channel outlet. At the point located at the rotor channel outlet there is an additional component with $\mathrm{f} \approx 800 \mathrm{~Hz}$, which was previously also noticed at point U6.

At points located after the rotor row, more additional components can be observed in the range of frequencies higher than $30 \mathrm{kHz}$ (e.g. 43.5 and $44 \mathrm{kHz}$ ). These components feature amplitudes of up to $150 \mathrm{~Pa}$ (the compo-

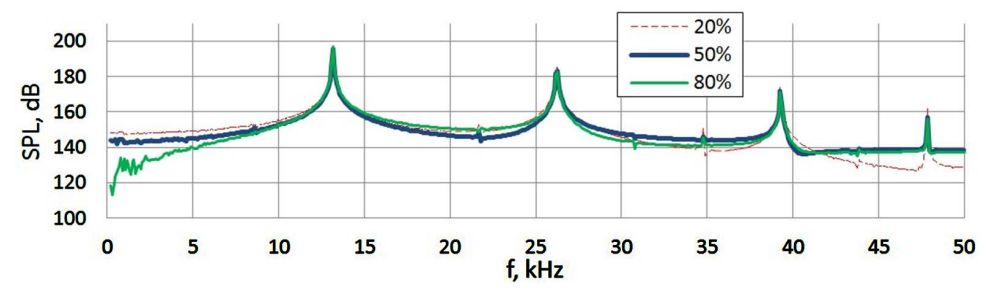

Figure 5. FFT analysis results for control points located before the rotor blade (points “Ro in b”).
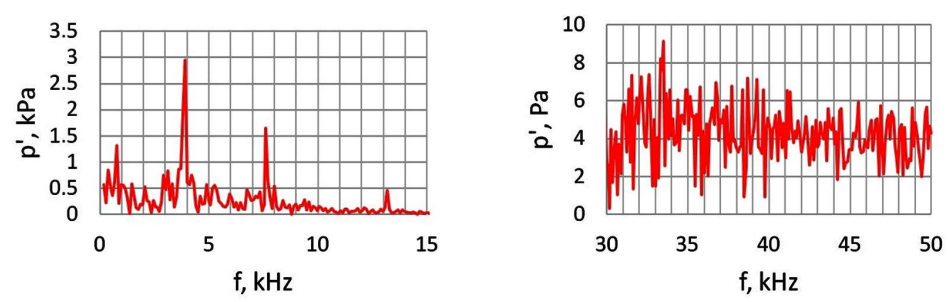

Figure 6. Results of the FFT analysis carried out for control point U6. 


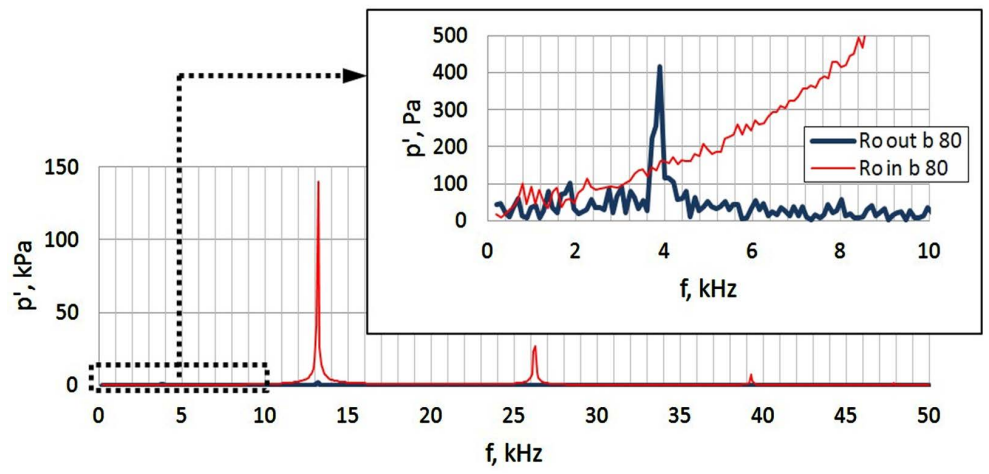

Figure 7. FFT analysis results for control points located before and after the rotor blade.

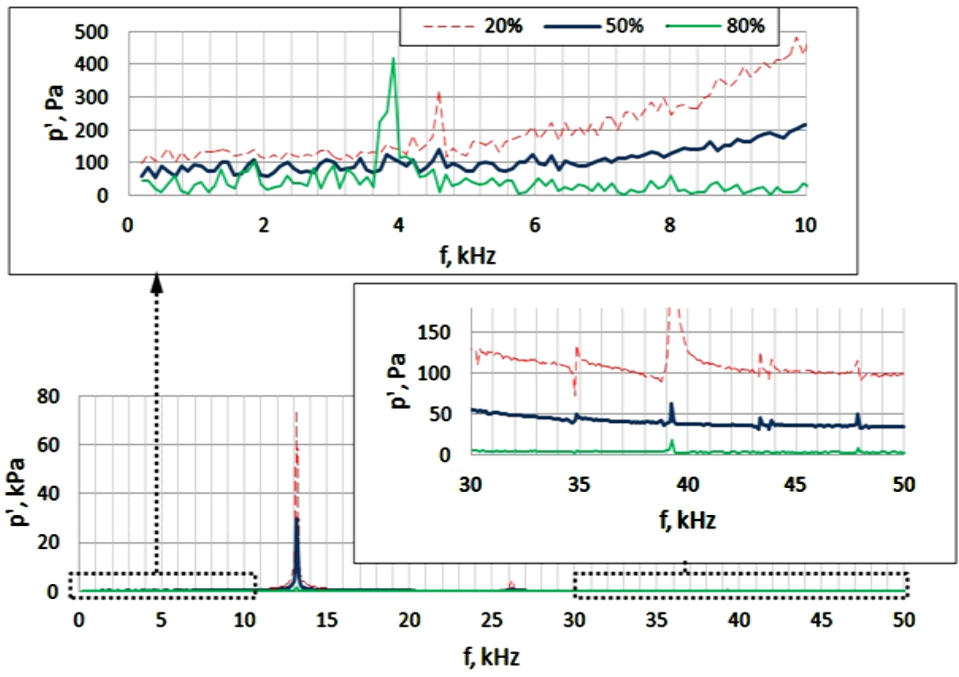

Figure 8. FFT analysis results for control points located after the rotor blade (points “Ro out b”).

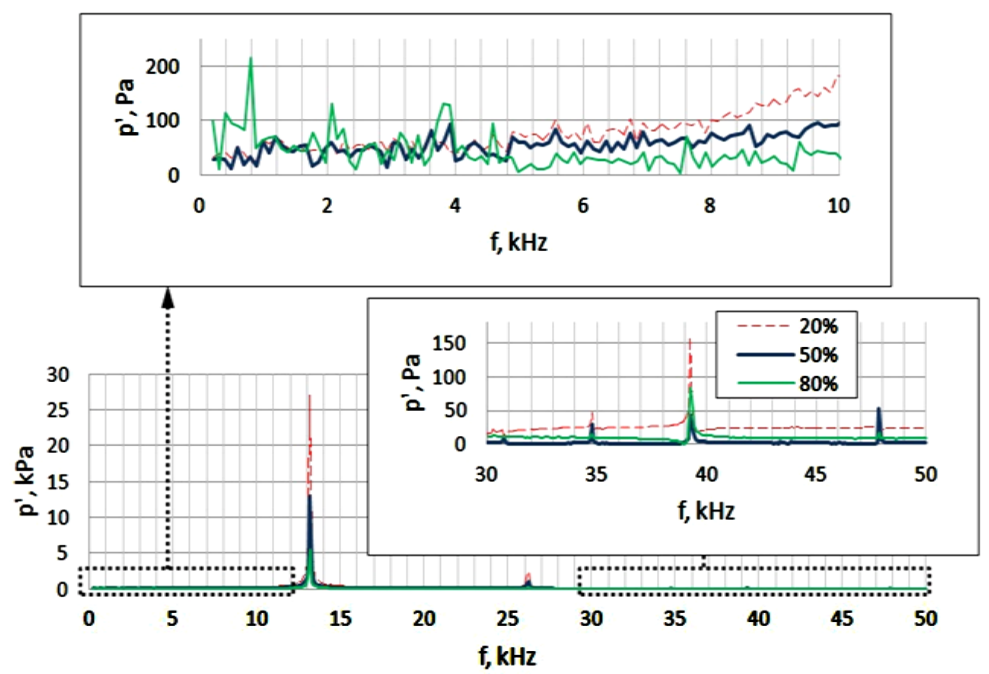

Figure 9. FFT analysis results for control points located at the rotor channel outlet (points "Ro out ch"). 


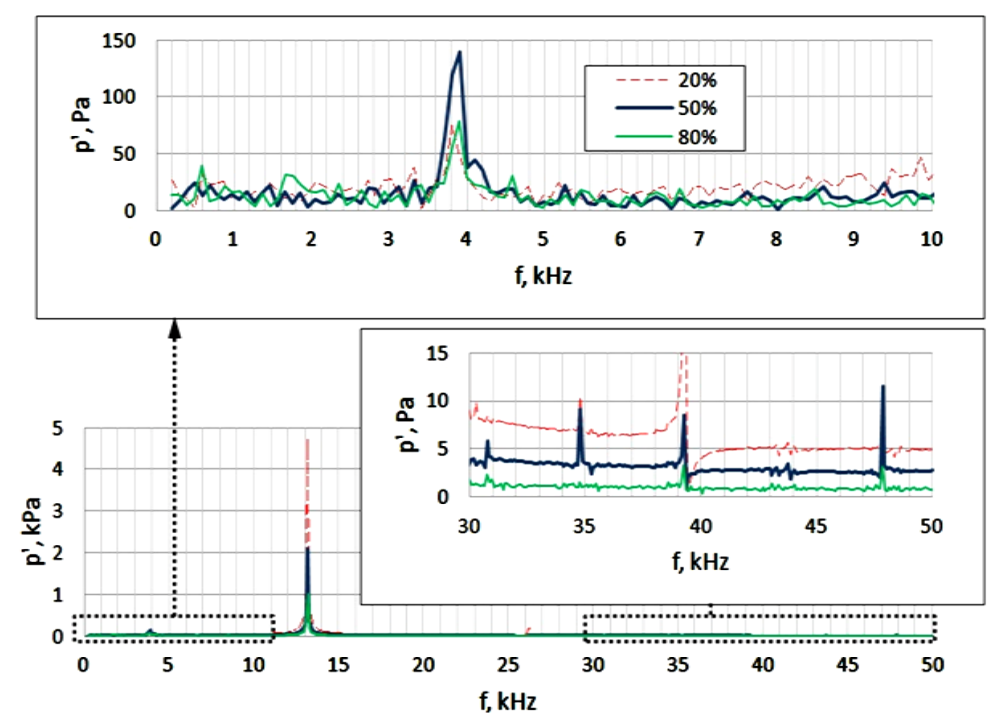

Figure 10. FFT analysis results for control points Ro o1 80, Ro o1 50, Ro 0130.

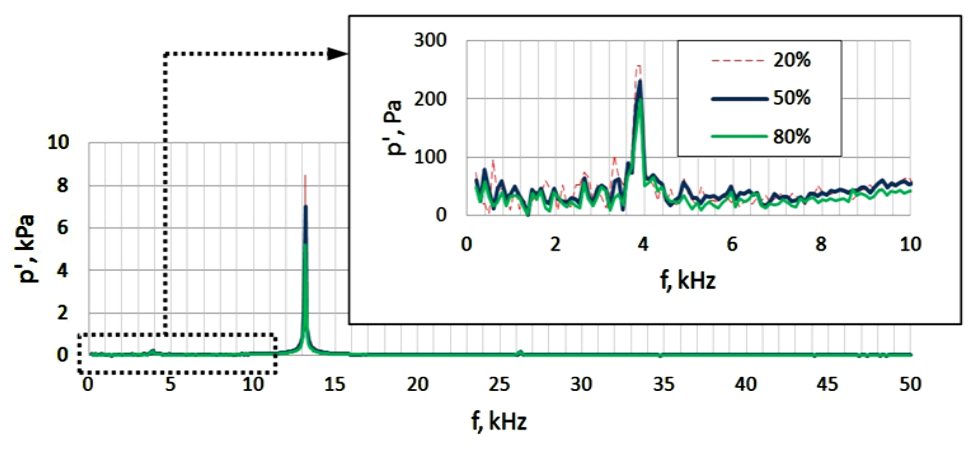

Figure 11. FFT analysis results for control points Ro o2 80, Ro o2 50, Ro o2 30.

nent being a VPF multiple ignored). They may prove the impact of the thermoacoustic effect on generation of acoustic waves in the area of the trailing edge because a very high temperature gradient occurs there between the medium flowing out of the blade channel and the medium in the aerodynamic wake. Amplitudes of components featuring frequencies attributed to the thermoacoustic effect are higher for points located in the blade wake. Only the strongest components are perceivable at the blade channel outlet.

\subsection{Points Located at the Rotor Extension}

For points located at the rotor extension, the distribution of amplitudes of the p' components depending on the channel height (Figure 10 and Figure 11) is the same as after the rotor. However, this distribution got partially blurred and at the channel extension the ratio of the p' amplitudes at $20 \%$ and $80 \%$ of the channel relative height does not exceed 5. The impact of the seal with its characteristic frequency $(\sim 3.9 \mathrm{kHz})$ can be observed at all points located at the channel extension. At the same time it should be noted that components featuring high frequencies are still present in the rotor extension, which probably results from the thermoacoustic effect.

\section{Conclusions}

This paper presents an analysis of the unsteady flow field in a turbine stage operating under a high load. The flow field unsteadiness was caused by a change in the mutual positioning of the stator and rotor blades and forced by aeroacoustic and thermoacoustic effects. The most common numerical method applied in fluid mechanics nowadays was employed for the calculations - the uRANS method available in the Ansys CFX software package. The analysis used the Adaptive-Scale Simulation (SAS) turbulence model, which belonged to the 
group of hybrid models. The main part of this paper is devoted to the analysis of the generation and propagation of pressure waves in a turbine stage. Comparing the results of the FFT analyses carried out for pressure fluctuations at selected points of the computational area, it is possible to make the following statements:

- The frequencies of the main harmonic components obtained from numerical computations subjected to the FFT analysis coincide with the frequencies of transition components resulting from theoretical dependences.

- The SAS model makes it possible to capture a large number of broadband noise components. These components are present especially in the range of low frequencies (up to $10 \mathrm{kHz}$ ), which is the acoustic range at the same time. Pressure fluctuations in the frequency range mentioned above result from the presence of large turbulence scales causing fluctuations in flow parameters.

- The SAS model also made it possible to capture noise components with relatively high amplitudes in the range of high frequencies. It is suspected that these components may result from the thermoacoustic effect induced by a high difference between the temperature of the blade wake right after the trailing edge and the temperature of the medium in its vicinity.

- The stator/rotor interface (keeping constant position with respect to the meshes) most probably did not interfere with the transmission of data concerning acoustic disturbances because significant and characteristic components generated in the seal area were also identified in the blade channel after the seal.

\section{Acknowledgements}

The authors would like to thank the Polish Ministry of Science and Higher Education for the financial support of the research project UMO-2011/01/B/ST8/03488.

\section{References}

[1] Denton, J.D. (1993) Loss Mechanisms in Turbomachines. Journal of Turbomachinery, 115, 621-656. http://dx.doi.org/10.1115/1.2929299

[2] Dykas, S., Wróblewski, W. and Lukowicz, H. (2007) Prediction of Losses in the Flow through the Last Stage of Low-Pressure Steam Turbine. International Journal for Numerical Methods in Fluids, 53, 933-945. http://dx.doi.org/10.1002/fld.1313

[3] Dykas, S., Wróblewski, W., Rulik, S. and Chmielniak, T. (2010) Numerical Method for Modeling of Acoustic Waves Propagation. Archives of Acoustics, 35, 35-48. http://dx.doi.org/10.2478/v10168-010-0003-7

[4] Dykas, S., Wróblewski, W. and Machalica, D. (2013) Numerical Analysis of the Losses in Unsteady Flow through Turbine Stage. Open Journal of Fluid Dynamics, 3, 252-260. http://dx.doi.org/10.4236/ojfd.2013.34031

[5] Świrydczuk, J. (2009) Three-Dimensional Unsteady Interaction of Vortex Structures in a Steam Turbine Rotor. Proceeding of the 8th European Conference on Turbomachinery, Fluid Dynamics and Thermodynamics, Graz, 23-27 March 2009, 1197-1206.

[6] Lampart, P. (2009) Investigation of Endwall Flows and Losses in Axial Turbines, Part I. Formation of Endwall Flows and Losses. Journal of Theoretical and Applied Mechanics, 47, 321-342.

[7] Egorov, Y., Menter, F.R., Lechner, R. and Cokljat, D. (2010) The Scale-Adaptive Simulation Method for Unsteady Turbulent Flow Predictions. Part 2: Application to Complex Flows. Flow Turbulence Combust, 85, 139-165. http://dx.doi.org/10.1007/s10494-010-9265-4

[8] Menter, F.R. and Egorov, Y. (2010) The Scale-Adaptive Simulation Method for Unsteady Turbulent Flow Predictions. Part 1: Theory and Model Description. Flow Turbulence Combust, 85, 113-138. http://dx.doi.org/10.1007/s10494-010-9264-5

[9] Moffit, T.P., Szanca, E.M. and Whitney, W.J. (1980) Design and Cold-Air Test of Single-Stage Uncooled Core Turbine with High Work Output, TP-1680, NASA.

[10] Whitney, W.J., Stabe, R.G. and Moffitt, T.P. (1980) Description of the Warm Core Turbine Facility and the Warm Annular Cascade Facility Recently Installed at NASA Lewis Research Center, Technical Report, NASA. Technical Memorandum 81562. 
Scientific Research Publishing (SCIRP) is one of the largest Open Access journal publishers. It is currently publishing more than 200 open access, online, peer-reviewed journals covering a wide range of academic disciplines. SCIRP serves the worldwide academic communities and contributes to the progress and application of science with its publication.

Other selected journals from SCIRP are listed as below. Submit your manuscript to us via either submit@scirp.org or Online Submission Portal.
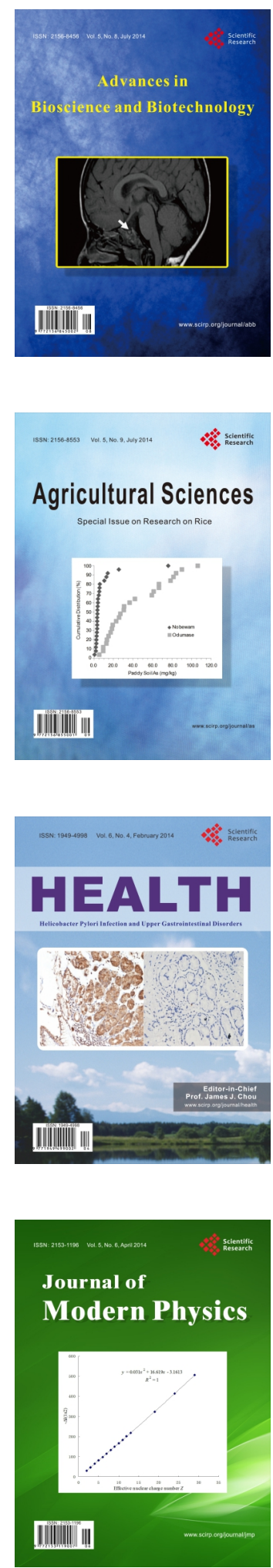
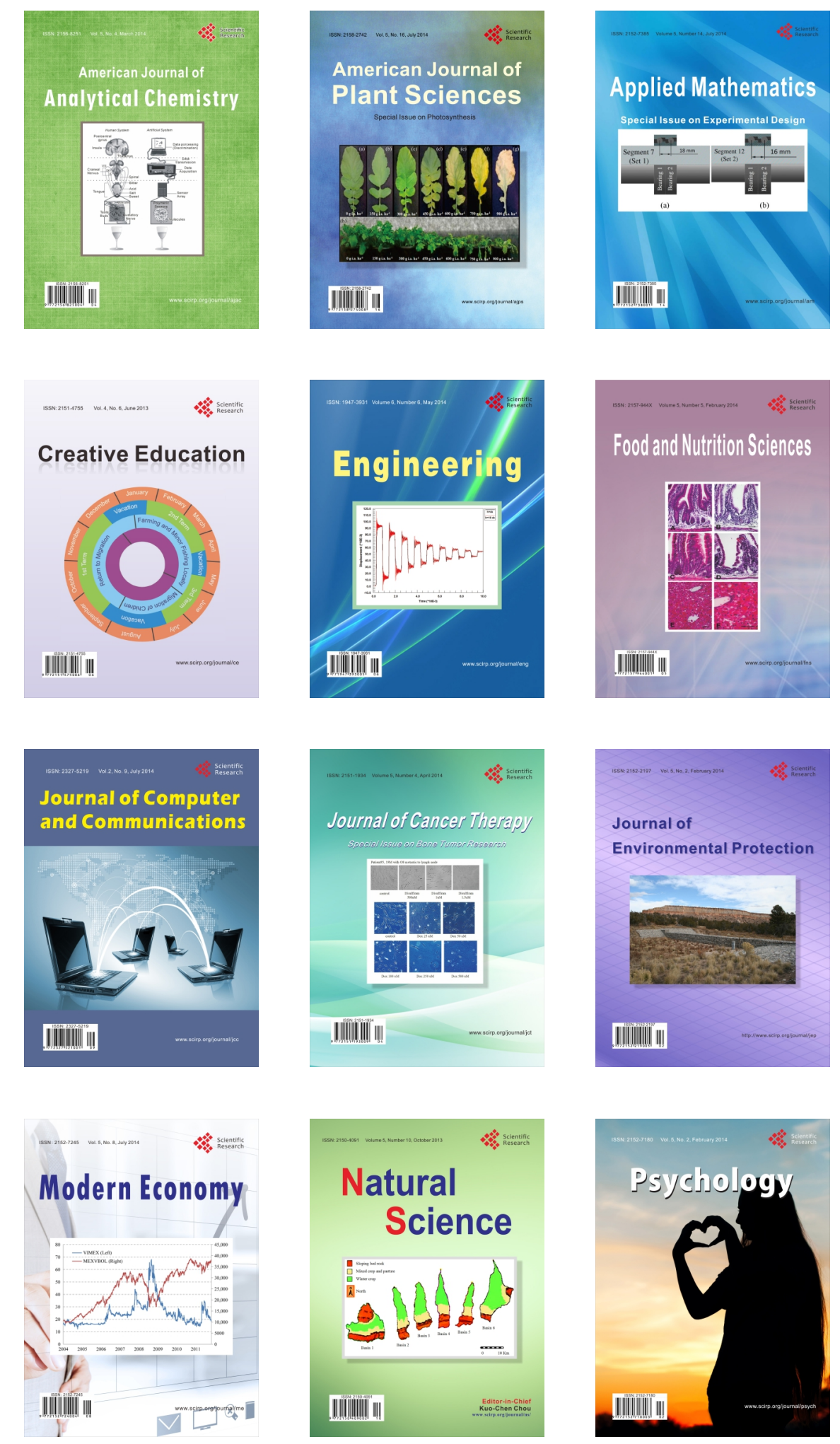